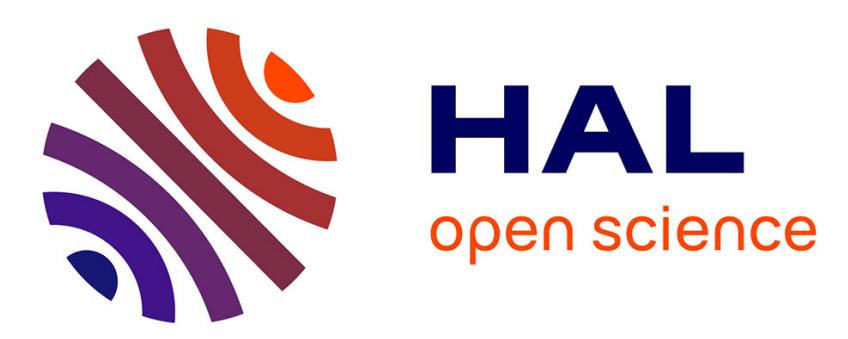

\title{
Analyse thermomécanique des lois de comportement par thermographie infrarouge
}

\author{
Andre Chrysochoos, J.-C. Chezeaux, H. Caumon
}

\section{To cite this version:}

Andre Chrysochoos, J.-C. Chezeaux, H. Caumon. Analyse thermomécanique des lois de comportement par thermographie infrarouge. Revue de Physique Appliquée, 1989, 24 (2), pp.215-225. 10.1051/rphysap:01989002402021500 . jpa-00246044

\section{HAL Id: jpa-00246044 https://hal.science/jpa-00246044}

Submitted on 1 Jan 1989

HAL is a multi-disciplinary open access archive for the deposit and dissemination of scientific research documents, whether they are published or not. The documents may come from teaching and research institutions in France or abroad, or from public or private research centers.
L'archive ouverte pluridisciplinaire HAL, est destinée au dépôt et à la diffusion de documents scientifiques de niveau recherche, publiés ou non, émanant des établissements d'enseignement et de recherche français ou étrangers, des laboratoires publics ou privés. 
Classification

Physics Abstracts

$46.20-46.30 \mathrm{~J}-46.30 \mathrm{R}$

\title{
Analyse thermomécanique des lois de comportement par thermographie infrarouge
}

\author{
A. Chrysochoos $\left({ }^{1}\right)$, J.-C. Chezeaux $\left({ }^{2}\right)$ et H. Caumon $\left({ }^{2}\right)$ \\ (1) Laboratoire de Mécanique Générale des Milieux Continus, U.S.T.L., Place Eugène Bataillon, 34060 \\ Montpellier, France \\ (2) Laboratoire de Mécanique et d'Acoustique, CNRS, 31 Chemin J. Aiguier, 13277 Marseille, France
}

(Reçu le 17 mars 1988, révisé le 20 septembre 1988 et le 24 octobre 1988, accepté le 8 novembre 1988)

\begin{abstract}
Résumé. - La connaissance des phénomènes dissipatifs et non dissipatifs associés aux processus quasistatiques de déformation, représente un atout supplémentaire important pour l'élaboration de loi thermomécanique de comportement. On décrit, ici, un dispositif expérimental, utilisant les techniques de thermographie infrarouge, et permettant d'observer les phénomènes thermiques et énergétiques durant la déformation d'un échantillon en traction simple. Après avoir rappelé la définition du bilan énergatique, dans le cas de matériaux élasto-plastiques, on relie la dissipation et l'énergie interne bloquée durant l'écrouissage, aux variables d'état. La puissance mécanique dissipée est évaluée continûment durant l'essai. On utilise pour cela, les images thermiques fournies par la caméra infra-rouge. On présente le dispositif expérimental, puis successivement, on décrit le modèle physique permettant de relier l'énergie dissipée aux cartes de température de surface ainsi que le protocole d'étalonnage. En fin d'article, on présente quelques résultats obtenus avec plusieurs matériaux et on évoque rapidement leur incidence sur la forme des lois de comportement.
\end{abstract}

\begin{abstract}
The knowledge of dissipative and non dissipative phenomena associated to quasi-static deformation processes, is a fundamental supplementary asset for determination of thermomechanical behaviour law. On depicts, in this paper, an experimental set-up, using infra-red technics, which allows to observe thermal and energetical phenomena during sample deformation. After restating the definition of the energy balance, in case of elastic-plastic materials, the relationship between the dissipation (or the stored energy of cold working) and the hardening state variables are recalled. The dissipation is continuously evaluated during monotonic tensile test. Numerized maps of surface temperature are used. The experimental arrangement is described ; then the physical model, which allows to relate the dissipation to the temperature signals, and the calibration method are successively introduced. Results on several materials are shown and their incidence on the behaviour law is succinctly mentioned.
\end{abstract}

\section{Introduction.}

Le processus de déformation entraîne, dans la plupart des cas, une modification du champ de température du matériau déformé. Ces phénomènes de couplage thermomécanique ont depuis longtemps été observés, et font, encore à l'heure actuelle, l'objet de nombreuses études expérimentales [1-10]. Le plus souvent, les capteurs de température utilisés sont des thermocouples collés ou soudés sur l'échantillon $[2,3,5]$, ou placés dans la cellule d'un calorimètre $[2,7,8]$. Depuis peu, les premiers dispositifs utilisant des capteurs infrarouges ont fait leur apparition sous forme de radiomètre (visée ponctuelle [9]), ou de caméra (champ [10]).
Le dispositif, présenté ici, utilise une caméra infrarouge équipée d'un système de numérisation du signal vidéo. Les images thermiques numérisées peuvent alors être traitées pour remonter localement aux sources mécaniques de chaleur. La réalisation d'un bilan énergétique permet d'accéder à la mesure de deux grandeurs physiques fondamentales pour le Mécanicien désireux d'établir des lois de comportement thermomécaniques : la dissipation et la variation d'énergie interne due aux modifications de la microstructure du matériau durant sa déformation, (énergie bloquée). Sur un plan théorique, en fait, ces phénomènes sont encore rarement pris en compte, à l'échelle du Milieu Continu, même si la 
Thermodynamique est désormais omniprésente dans la construction des modèles [11-13].

\section{Situation du problème.}

Dans le cas d'un matériau élastoplastique, le bilan énergétique peut s'écrire de la manière suivante [14] :

$$
W_{\text {ext }}=W_{\mathrm{e}}+W_{\mathrm{d}}+W_{\mathrm{b}}
$$

où $W_{\text {ext }}$ représente l'énergie mécanique fournie pour déformer le matériau, $W_{\mathrm{e}}$ l'énergie élastique réversible, $W_{\mathrm{d}}$ l'énergie dissipée et $W_{\mathrm{b}}$ l'énergie interne bloquée durant l'écrouissage.

Ce bilan est illustré sur la figure 1 , dans le cas d'un cycle charge-décharge. Le terme complémentaire $W_{\text {is }}$ est le terme isentropique associé aux effets thermoélastiques. L'analyse du bilan énergétique complet du cycle montre que la variation d'énergie interne $\left.\Delta E\right|_{0} ^{B}$ correspond à la variation d'énergie bloquée durant l'écrouissage :.

$$
\begin{aligned}
& \left.\Delta E\right|_{0} ^{A}=W_{\text {ext }}+W_{\text {is }}-W_{\mathrm{d}} \\
& \left.\Delta E\right|_{A} ^{B}=-W_{\text {is }}-W_{\mathrm{e}} \\
& \left.\Delta E\right|_{0} ^{B}=W_{\mathrm{b}} .
\end{aligned}
$$

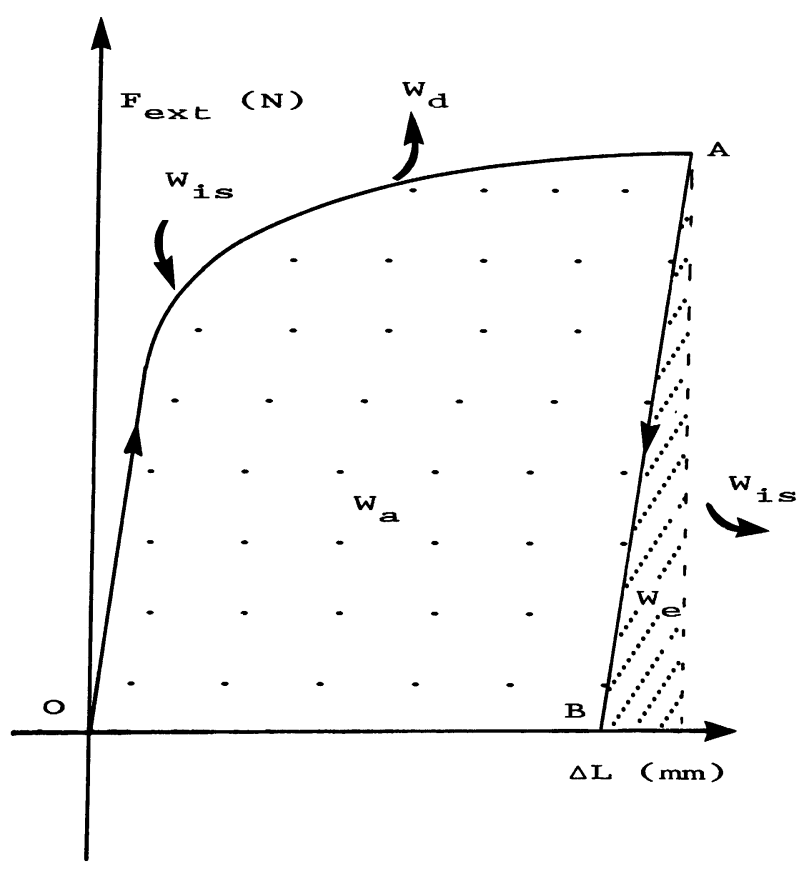

Fig. 1. - Bilan énergétique pour un cycle chargedécharge.

[Energy balance for a load-unload cycle.]

En utilisant les résultats classiques de la Thermodynamique des Milieux Continus [11, 12], on définit la dissipation intrinsèque, puissance associée à $W_{\mathrm{d}}$ :

$$
D_{1}=\sigma: D-\rho \frac{\partial \psi}{\partial \alpha_{j}} \dot{\alpha}_{j}
$$

où $\sigma$ est le tenseur des contraintes de Cauchy et $D$ le tenseur vitesse de déformation. Les termes en $\rho \frac{\partial \psi}{\partial \alpha_{j}} \dot{\alpha}_{j}$ représentent la puissance associée aux variables d'état $\left(\alpha_{j}\right), j=1,2, \ldots, n$, intervenant dans l'énergie libre de Helmholtz $\psi\left(T, \alpha_{j}\right), \alpha_{0}=T$ étant la température absolue et $\rho$ la masse volumique. En général, dans le cas de matériaux élastoplastiques, la variable d'état $\alpha_{1}$ est un tenseur $\varepsilon_{\mathrm{e}}$ récapitulant les déformations élastiques. Les variables d'état $\left(\alpha_{j}\right), j=2, \ldots, n$, caractérisent l'état d'écrouissage du matériau, et les termes $\rho \frac{\partial \psi}{\partial \alpha_{j}}$, $j=2, \ldots, n$, peuvent être associés aux contraintes internes dues aux modifications microstructurales : augmentation de la densité de dislocations, modification de la taille des cellules, etc.

$\mathrm{Si}$ on suppose que les (petites) variations de température, dues au processus d'écrouissage, ne modifient pas l'état d'écrouissage du matériau (pas de phénomène de restauration induite par la dissipation),

$$
\rho \frac{\partial^{2} \psi}{\partial \alpha_{j} \partial T} \dot{\alpha}_{j} \approx 0, \quad j=2, \ldots, n
$$

la dissipation et le terme isentropique sont alors les seuls termes sources d'origine mécanique présents au second membre de l'équation de la chaleur:

$$
\rho C_{\alpha} \dot{\theta}+\operatorname{div}(q)=D_{1}+\rho T \frac{\partial^{2} \psi}{\partial \theta \partial \varepsilon_{\mathrm{e}}} \dot{\varepsilon}_{\mathrm{e}}=\dot{w}_{\mathrm{ch}}
$$

où $\theta=T-T_{\mathrm{a}}\left(T_{\mathrm{a}}\right.$ : champ de température homogène à l'équilibre), $q$ est le vecteur courant de chaleur échangée avec le milieu extérieur, (loi de Fourier isotrope),

$$
q=-k \operatorname{grad}(\theta)
$$

et où on suppose que le terme volumique de source de chaleur échangée avec l'extérieur est indépendant du temps. La capacité calorifique $C_{\alpha}$ et le coefficient de conduction $k$ sont supposés constants, indépendants de l'état d'écrouissage du matériau, et de la température.

\section{Mesures mécaniques.}

Dans le cas d'essais en traction simple, les états de contrainte et de déformation peuvent être considérés comme homogènes dans toute la partie utile de l'éprouvette. Ainsi, en enregistrant durant l'essai les signaux force et allongement, on peut évaluer la composante $\sigma$ dans l'axe de chargement du tenseur 
des contraintes, ainsi que les composantes correspondantes $\varepsilon_{\mathrm{e}}$ et $\varepsilon_{\mathrm{p}}$ du tenseur des déformations élastiques et plastiques.

En supposant le comportement élastique linéaire isotrope, ces grandeurs sont utilisées pour calculer l'énergie « anélastique » $\left(W_{\mathrm{a}}=W_{\text {ext }}-W_{\mathrm{e}}\right)$ et l'énergie isentropique (cf. [20], p. 177) :

$$
\begin{aligned}
W_{\mathrm{a}}=W_{\mathrm{d}} & +W_{\mathrm{b}}=V_{0} \int_{0}^{\varepsilon_{p}(t)} \sigma \mathrm{d} \varepsilon_{p} \\
-W_{\mathrm{is}}=\int_{0}^{t} \int_{\Omega(\tau)} \rho T & \frac{\partial^{2} \psi}{\partial \theta \partial \varepsilon_{\mathrm{e}}} \dot{\varepsilon}_{\mathrm{e}} \mathrm{d} x \mathrm{~d} \tau \cong \\
& \cong \int_{0}^{t} \int_{\Omega(\tau)} \lambda T_{\mathrm{a}} \operatorname{tr}(\dot{\sigma}) \mathrm{d} x \mathrm{~d} \tau \\
& =V_{0} \lambda T_{\mathrm{a}}(\sigma(t)-\sigma(0))
\end{aligned}
$$

$\lambda$ étant le coefficient de dilatation thermique du matériau, $V_{0}$ représentant le volume de la partie utile de l'éprouvette $\left(V_{0}=8,4 \times 10^{-6} \mathrm{~m}^{3}\right)$.

\section{Description du dispositif infrarouge.}

L'utilisation des techniques infrarouges en Mécanique des Solides est récente. Pour illustrer la diversité et le caractère prometteur des applications de ces nouvelles techniques à la Mécanique, on renvoie le lecteur intéressé, à la liste des références citées dans [15].

L'élément essentiel de ce dispositif est bien évidemment la caméra infrarouge (commercialisée par la firme AGA), munie de son système de numérisation du signal vidéo (développé par le L.M.A.). L'ensemble est interfacé avec un calculateur et permet l'enregistrement d'images thermiques numérisées. Cette numérisation du signal, permet moyennant un traitement numérique adéquat d'améliorer considérablement le rapport signal sur bruit.

3.1 LA MACHINE D'ESSAI. - Sur la machine d'essai mécanique (INSTRON), on met en place une enceinte métallique, permettant de réaliser des essais sous vide primaire $\left(10^{-2} \mathrm{mbar}\right)$. En manipulant sous vide, on minimise les fuites thermiques par convection et on améliore le rapport signal sur bruit. L'enceinte est munie de soufflets élastiques qui permettent les déplacements de la traverse. Un hublot mobile sur la face arrière permet le montage et démontage de l'éprouvette de traction et la mise en place de l'extensomètre sur la partie utile de l'échantillon. Sur la face avant, la caméra vient prendre place devant un hublot de fluorine qui assure une bonne transmission du rayonnement.

3.2 LA CAMÉRA INFRAROUGE. - La caméra infrarouge permet de transformer l'énergie du rayonnement (infrarouge) émis par une cible en un signal électrique, (voir, par exemple [15, 17] pour plus de détails).
L'éprouvette de traction (la cible), recouverte d'une fine couche de peinture spéciale à fort coefficient d'émissivité, est considérée comme un corps gris. L'énergie du rayonnement électromagnétique émis localement par l'échantillon peut alors être théoriquement reliée, en vertu des lois de Planck et de Stefan-Boltzmann, à la température du corps.

En pratique, la relation entre signal vidéo et température de surface de l'échantillon n'est pas aussi «simple ». Le rayonnement émis par le matériau traverse un certain nombre de milieux (atmosphère, optique) avant d'être analysé par la caméra. De plus des rayonnements parasites, provenant de l'environnement (enceinte, mors, etc.), viennent bruiter l'information saisie par la caméra et rendent encore plus délicats les problèmes de métrologie.

Aussi, les images thermiques numérisées, doiventelles subir un certain nombre de pré-traitements avant de pouvoir être exploitées. Le détail du protocole d'étalonnage et de reconstitution des images thermiques est donné dans [16]. Pour illustrer les performances du dispositif, quelques chiffres significatifs peuvent être cités. L'image thermique numérisée représente une matrice $128 \times 200$ points en température ; la résolution spatiale est dans notre cas d'environ $0,12 \times 10^{-3} \mathrm{~m}$; sur la gamme la plus sensible de la caméra, un étalonnage du signal en température a donné un écart de $0,027^{\circ} \mathrm{C}$ par niveau informatique (i.e. par bit). La vitesse d'acquisition est d'environ une image complète par seconde.

On présente quatre images thermiques d'un échantillon de duralumin en cours de déformation, (cf. planches 1 à 4). La courbe (Fig. 2) illustre l'évolution de la température moyenne de la surface $S$ définie en 3.3.1.

3.3 DÉTERMinATION DE LA SOURCE MÉCANIQUE DE CHALEUR. - Durant l'essai mécanique, la caméra enregistre l'évolution du champ de température sur une partie de la surface de l'échantillon. On utilise ces cartes de température pour évaluer, par l'intermédiaire de l'équation de diffusion de la chaleur, les sources de chaleur d'origine mécanique regroupées au second membre ; le premier membre pouvant s'écrire sous la forme d'un opérateur différentiel appliqué à la température.

Si on admet la loi linéaire de Fourier pour la conduction de la chaleur dans le matériau, supposé thermiquement isotrope, cette équation s'écrit en tout point :

$$
\begin{aligned}
& \rho C_{\alpha}\left[\frac{\partial \theta}{\partial t}+u \cdot \operatorname{grad}(\theta)\right]- k \Delta \theta= \\
&=D_{1}-\dot{w}_{\mathrm{is}}=\dot{w}_{\mathrm{ch}}
\end{aligned}
$$

où le terme entre crochets représente la dérivée particulaire de la température, $u$ étant la vitesse de la particule.

Un calcul d'ordre de grandeur montre que le 


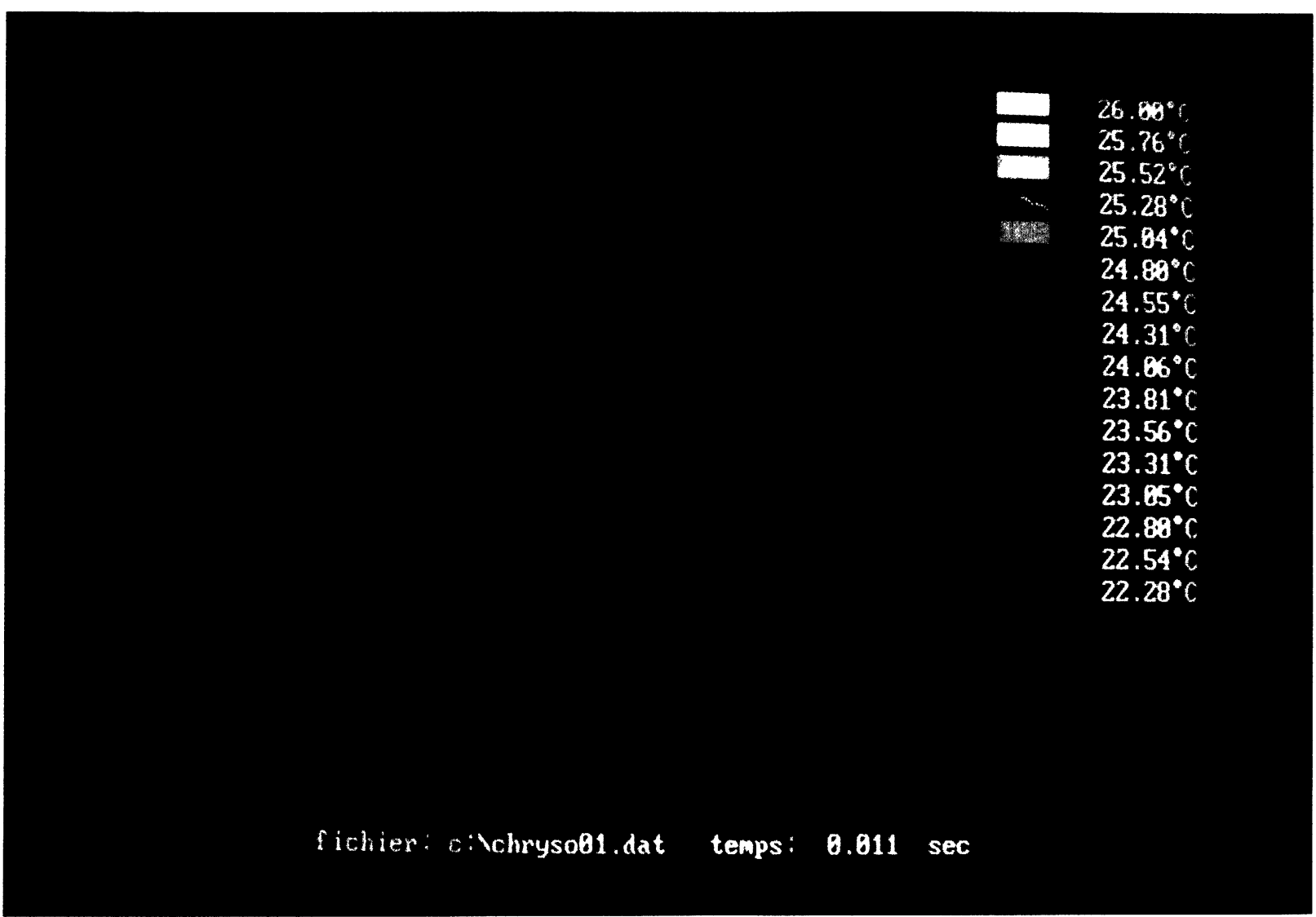

Planche 1. - L'éprouvette de duralumin est recouverte d'une fine couche de peinture noire et se détache du fond (à faible coefficient d'émissivité). L'éprouvette est à l'équilibre thermique.

[The AU4G sample is coated with a thin black painting and stands out against the background (with short emissivity coefficient). The sample is in thermal equilibrium.]

terme convectif $u \cdot \operatorname{grad}(\theta)$ reste négligeable dans le cas d'essai en traction simple (densité homogène de chaleur), sur des matériaux métalliques (bonne diffusivité thermique, peu de gradient), lors de processus quasi-statiques (très faibles vitesses).

L'épaisseur de l'éprouvette de traction étant petite, la température varie peu suivant cette direction; on peut donc se ramener formellement à l'étude d'un milieu bidimensionnel, en intégrant (10) dans l'épaisseur de l'éprouvette de traction :

$\rho C_{\alpha} \frac{\partial \bar{\theta}}{\partial t}-k\left(\frac{\partial^{2} \bar{\theta}}{\partial x^{2}}+\frac{\partial^{2} \bar{\theta}}{\partial y^{2}}\right)-\frac{k}{e}\left[\frac{\partial \theta}{\partial z}\right]_{0}^{e}=\dot{w}_{\mathrm{ch}}$

où :

$$
\overline{\theta(t, x, y)}=\frac{1}{e} \int_{0}^{e} \theta(t, x, y, z) \mathrm{d} z
$$

représente la température moyenne dans l'épaisseur.

Le terme entre crochets représente le flux de chaleur perdu par les faces de l'éprouvette. Sous vide partiel, ces pertes se font presque exclusivement par rayonnement. La loi de Stefan-Boltzmann fournit alors une expression du flux de chaleur :

$$
-k\left[\frac{\partial T}{\partial z}\right]_{0}^{e}=2 \varepsilon_{\mathrm{m}} \sigma_{\mathrm{E}} \bar{T}^{4}
$$

où $\varepsilon_{\mathrm{m}}$ est l'émissivité du corps considéré comme gris et $\sigma_{\mathrm{E}}$ la constante de Stefan-Boltzmann ; en linéarisant (12) au voisinage de la température d'équilibre, l'équation (11) devient :

$$
\begin{aligned}
\rho C_{\alpha} \frac{\partial \bar{\theta}}{\partial t}-k\left(\frac{\partial^{2} \bar{\theta}}{\partial x^{2}}+\frac{\partial^{2} \bar{\theta}}{\partial y^{2}}\right)+ & \\
+8 & \frac{\varepsilon_{\mathrm{m}} \sigma_{\mathrm{E}}}{e} T_{\mathrm{a}}^{3} \bar{\theta}=\dot{w}_{\mathrm{ch}} .
\end{aligned}
$$

3.3.1 Estimation du Laplacien. - Lors de l'estimation du Laplacien à partir des données en température, le bruit numérique est considérablement amplifié (dérivée seconde de données déjà bruitées). Les techniques classiques de filtrage et d'estimation d'opérateur (méthodes convolutives), ont été éprouvées. Ces méthodes ont été utilisées avec succès par [15] sur des matériaux peu conducteurs, lors de phénomènes stationnaires, pour lesquels un débruitage efficace des cartes thermiques pouvait être fait, par cumul de l'information dans le temps. Dans le 


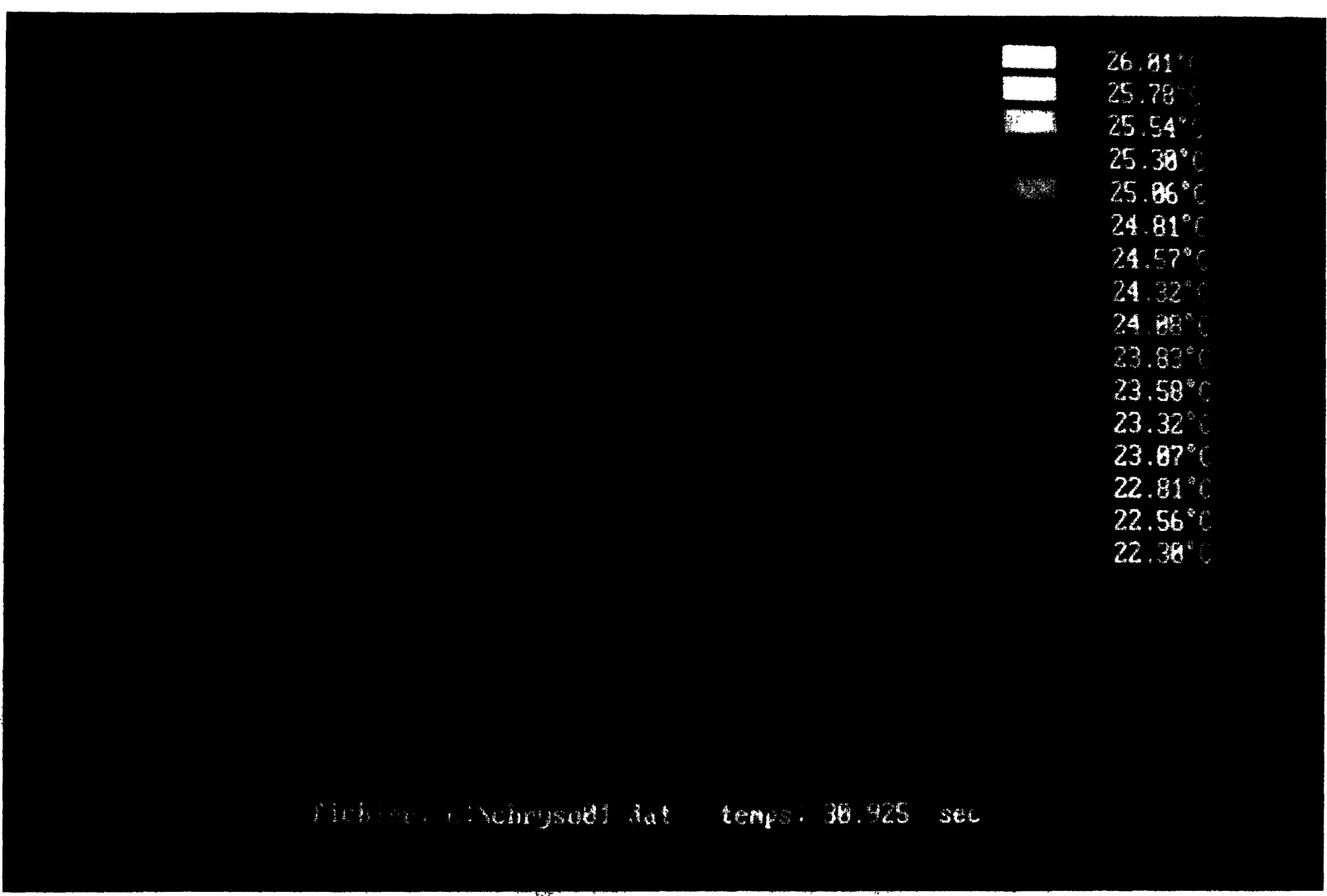

Planche 2. - Au bout de $30 \mathrm{~s}$, la contrainte limite élastique est quasiment atteinte ; l'éprouvette se refroidit (d'environ $0,2^{\circ} \mathrm{C}$ ) et se confond maintenant avec l'arrière plan.

[At time $t=30 \mathrm{~s}$, the yield stress is almost reached; the sample is cooling, (around $0.2^{\circ} \mathrm{C}$ ) and cannot be more distinguished from the background.]

cadre de nos essais, celles-ci n'ont pas donné satisfaction car le rapport bruit thermique sur résolution spatiale (densité spatiale d'information) est trop important, et nous n'avons pu trouver de filtres classiques (fenêtre de Hamming, Gauss, fonction porte, etc.), qui éliminent efficacement le bruit, sans du même coup, «écraser » totalement les gradients thermiques.

C'est pourquoi, afin d'éviter toute estimation locale du Laplacien, on choisit de moyenner (13) sur un petit pavé $S$ d'observation, centré sur la partie utile de l'éprouvette, de manière à faire disparaître tous les termes du second ordre :

$$
\frac{\partial \overline{\bar{\theta}}}{\partial t}-\frac{\beta}{S} \int_{\partial S} \operatorname{grad}(\bar{\theta}) \cdot n \mathrm{~d}(\partial S)+\frac{\overline{\bar{\theta}}}{\tau_{\mathrm{r}}}=\frac{\dot{w}_{\mathrm{ch}}}{\rho C_{\alpha}}
$$

où :

$$
\overline{\overline{\overline{\theta(t)}}}=\frac{1}{S} \iint_{S} \bar{\theta}(t, x, y) \mathrm{d} x \mathrm{~d} y
$$

est la température moyenne du petit volume $e \times S$ d'observation et où l'on a posé :

$$
\beta=\frac{k}{\rho C_{\alpha}}, \frac{1}{\tau_{\mathrm{r}}}=8 \frac{\sigma_{\mathrm{E}} \varepsilon_{\mathrm{m}}}{e \rho C_{\alpha}} T_{\mathrm{a}}^{3} .
$$

Le deuxième terme du premier membre de (14), correspond aux fuites par conduction à la frontière $\partial S \mathrm{du}$ domaine $S$. On prolonge l'hypothèse de linéarité des fuites par rayonnement, faite précédemment, aux fuites par conduction, de sorte que :

$$
\frac{\beta}{S} \int_{\partial S} \operatorname{grad}(\bar{\theta}) \cdot n \mathrm{~d}(\partial S)=\frac{\overline{\bar{\theta}}}{\tau_{\mathrm{c}}}
$$

où $\tau_{\mathrm{c}}$ représente la constante de temps caractéristique des fuites par conduction.

En rapprochant alors (14) et (16), l'équation de la chaleur prend alors la forme suivante :

$$
\frac{\partial \overline{\bar{\theta}}}{\partial t}+\frac{\overline{\bar{\theta}}}{\tau_{\text {th }}}=\frac{\dot{w}_{\mathrm{ch}}}{\rho C_{\alpha}}
$$

avec $\tau_{\text {th }}=\frac{\tau_{\mathrm{c}} \tau_{\mathrm{r}}}{\tau_{\mathrm{c}}+\tau_{\mathrm{r}}}$ constante de temps caractéristique des fuites thermiques au niveau du volume observé $e \times S$.

Il est intéressant de remarquer dans le cas où $S=4 a b$ que :

$$
\frac{\beta}{S} \int_{S} \Delta \bar{\theta} \mathrm{d} S=\frac{1}{\tau_{c} S} \int_{-a}^{+a} \int_{+b}^{+b} \bar{\theta}(x, y) \mathrm{d} x \mathrm{~d} y
$$




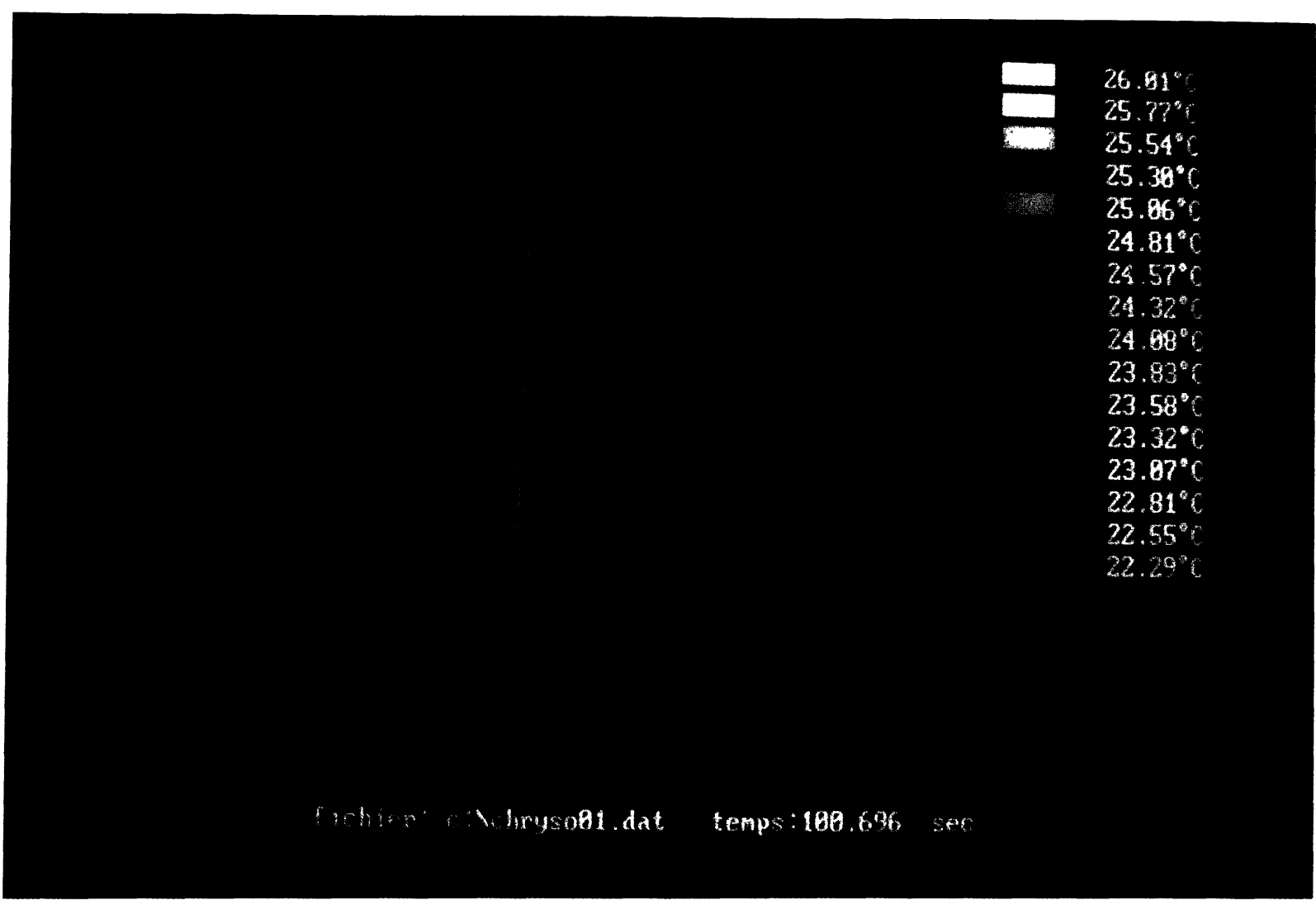

Planche 3. - Une fois la limite élastique dépassée, la dissipation combat l'effet de refroidissement dû au terme source isentropique. L'éprouvette commence à s'échauffer.

[When the stress is upper than the yield stress, then the dissipation fights against the thermoelastic effect. The sample is overheating.]

$$
\begin{aligned}
& \frac{\beta}{S} \int_{-a}^{+a} \int_{-b}^{+b} \Delta \bar{\theta} \mathrm{d} x \mathrm{~d} y= \\
&=\frac{1}{\tau_{\mathrm{c}} S} \int_{-\infty}^{+\infty} \int_{-\infty}^{+\infty} \theta\left(x^{\prime}, y^{\prime}\right) \\
& \times \Pi_{\mathrm{a}}\left(-x^{\prime}\right) \Pi_{\mathrm{b}}\left(-y^{\prime}\right) \mathrm{d} x^{\prime} \mathrm{d} y^{\prime}
\end{aligned}
$$

où

$$
\underset{\alpha>0}{\Pi_{\alpha}(\beta)}=\left\{\begin{array}{lll}
1 & \text { si } & |\beta|<\alpha \\
0 & \text { si } & |\beta|>\alpha
\end{array}\right.
$$

de sorte que (16) apparaît comme un cas particulier des méthodes de filtrage convolutif où le filtre appliqué à la température est de la forme :

$$
\xi_{a, b}\left(\nu_{x}, \nu_{y}\right)=4 \frac{\sin a \nu_{x}}{\nu_{x}} \cdot \frac{\sin b \nu_{y}}{\nu_{y}}
$$

Le pavé d'observation choisi est un rectangle d'environ $2 \mathrm{~mm}$ de large sur $20 \mathrm{~mm}$ de long. Sachant que l'ordre de grandeur de la constante est de plusieurs dizaines de secondes dans le cas des matériaux métalliques étudiés, et en rappelant que l'erreur due au bruit décroît grosso modo, en $N^{1 / 2}$, où $N$ est le nombre d'informations, on peut estimer l'erreur commise en évaluant le Laplacien :

$$
\sigma_{\Delta \theta}=\frac{\delta \theta}{\sqrt{N} \tau_{c}} \approx 5 \times 10^{-5}{ }^{\circ} \mathrm{C} . \mathrm{s}^{-1}
$$

avec les valeurs numériques suivantes :

$$
\delta \theta \approx 0,027^{\circ} \mathrm{C} ; \quad N=2700 ; \quad \tau_{\mathrm{c}} \approx 10 \mathrm{~s} .
$$

3.3.2 Estimation du terme instationnaire. - La dérivée de la température par rapport au temps est plus aisée. Une évaluation par différences finies symétriques est suffisante :

$$
\frac{\partial \overline{\bar{\theta}}}{\partial t} \cong \frac{\overline{\bar{\theta}}(t+h)-\overline{\bar{\theta}}(t-h)}{2 h}
$$

avec $h$, intervalle de temps entre deux enregistrements.

Dans les mêmes conditions que précédemment, le niveau de bruit sur ce terme est de l'ordre de $10^{-4}{ }^{\circ} \mathrm{C} . \mathrm{s}^{-1}$. 


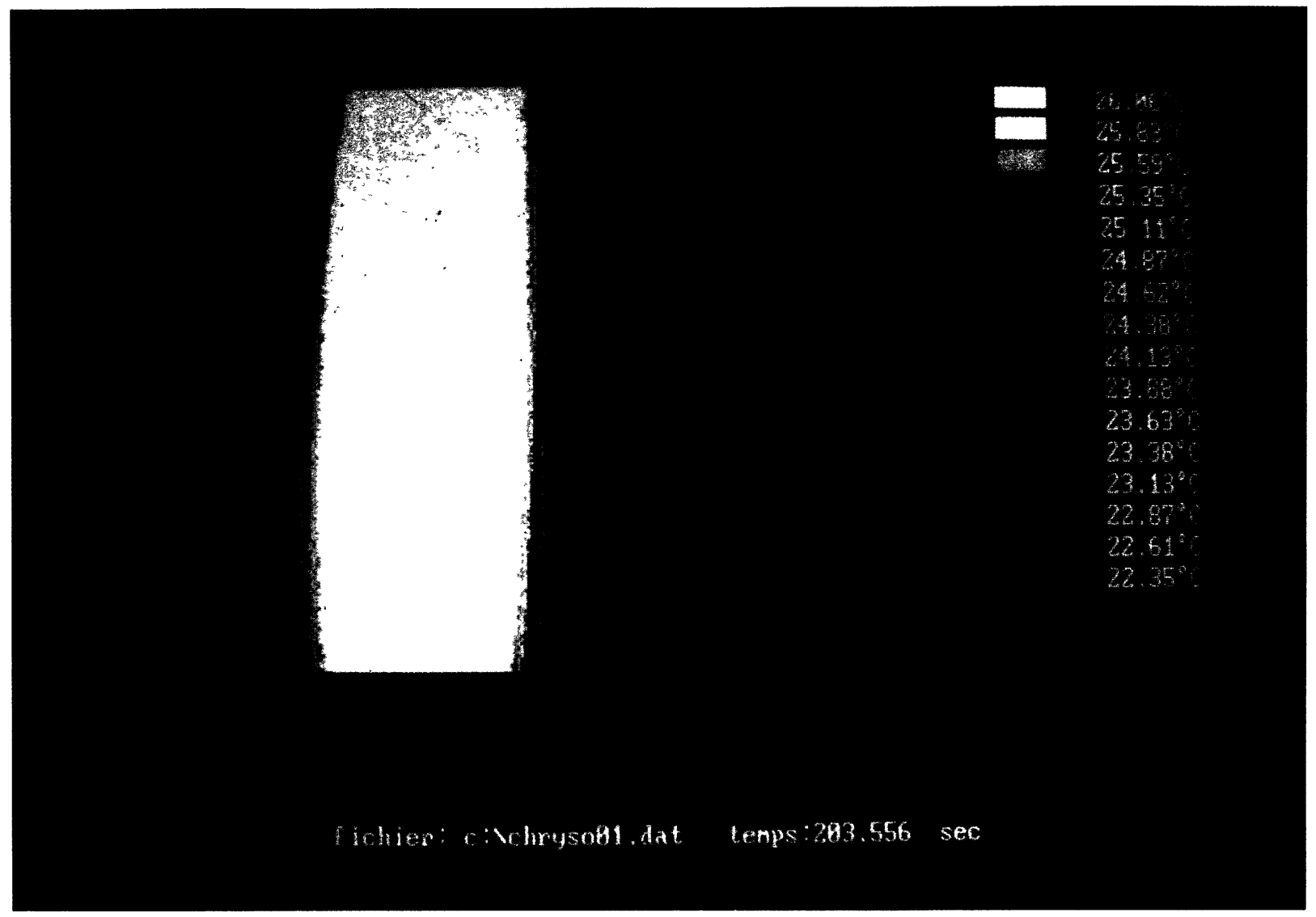

Planche 4. - Pour une déformation de $10 \%$, à $\dot{\varepsilon} \approx 10^{-4} \mathrm{~s}^{-1}$, l'échauffement en fin d'essai de traction est d'environ $3^{\circ} \mathrm{C}$ pour un duralumin. Il est un peu plus important pour l'acier au carbone (4 à $\left.5^{\circ} \mathrm{C}\right)$, et l'inox $\left(5\right.$ à $\left.6^{\circ} \mathrm{C}\right)$.

[For strain at $10 \%$ and for strain rate $\dot{\varepsilon} \approx 10^{-4} \mathrm{~s}^{-1}$, the heating at the end of test reaches around $3^{\circ} \mathrm{C}$, in the case of AU4G sample. It is a little bit more important in case of carbon steel $\left(4\right.$ to $\left.5^{\circ} \mathrm{C}\right)$ and stainless steel $\left(5\right.$ to $\left.6^{\circ} \mathrm{C}\right)$.]

\subsection{EtAlonNAGes.}

3.4.1 Les fichiers de référence. - L'information fournie par la caméra est bruitée. Les origines de ce bruit proviennent de rayonnements parasites générés par le milieu extérieur (enceinte, atmosphère, caméra), de l'électronique (dérives), et numérique (convertisseur 8 bits).

Si les phénomènes de dérive peuvent être considérés comme négligeables, $P$. Bremond [16] montre qu'il est absolument nécessaire, dans chaque manipulation, d'enregistrer, au préalable, une image thermique moyenne de référence, afin de minimiser, par soustraction, l'importance des aberrations thermiques stationnaires provenant des réflections parasites.

3.4.2 Etalonnage du signal vidéo. - L'étalonnage consiste à établir la correspondance entre un niveau thermique numérisé, enregistré par le calculateur, et l'écart de température qui le produit.

Les courbes d'étalonnage, fournies par le constructeur de la caméra, montrent que dans le cadre de nos expériences (faibles variations de température), la tension du signal est proportionnelle à la température. L'étalonnage peut alors se faire en deux points de mesure. Il a été réalisé par [16] ; sur la gamme la plus sensible de la caméra, on a trouvé un écart de $0,027^{\circ} \mathrm{C}$ par niveau informatique, pour une résolution spatiale de $0,12 \mathrm{~mm}$.

3.4.3 Détermination de $\tau_{\mathrm{th}}$. - L'hypothèse (16) de linéarité des fuites a été soigneusement contrôlée [18]. Ce contrôle a été réalisé :

- en observant le retour à l'équilibre thermique d'éprouvettes initialement préchauffées par effet Joule, par l'intermédiaire de résistances électriques planes de faible capacité calorifique ;

- en jouant sur le refroidissement et le réchauffement isentropique dûs au couplage thermoélastique, et en comparant la réponse thermique observée avec le dispositif à celle calculée théoriquement, (cf. Fig. 3) ;

- en étudiant la solution analytique du champ de température, solution d'un problème dont les conditions limites sont proches des conditions expérimentales. 


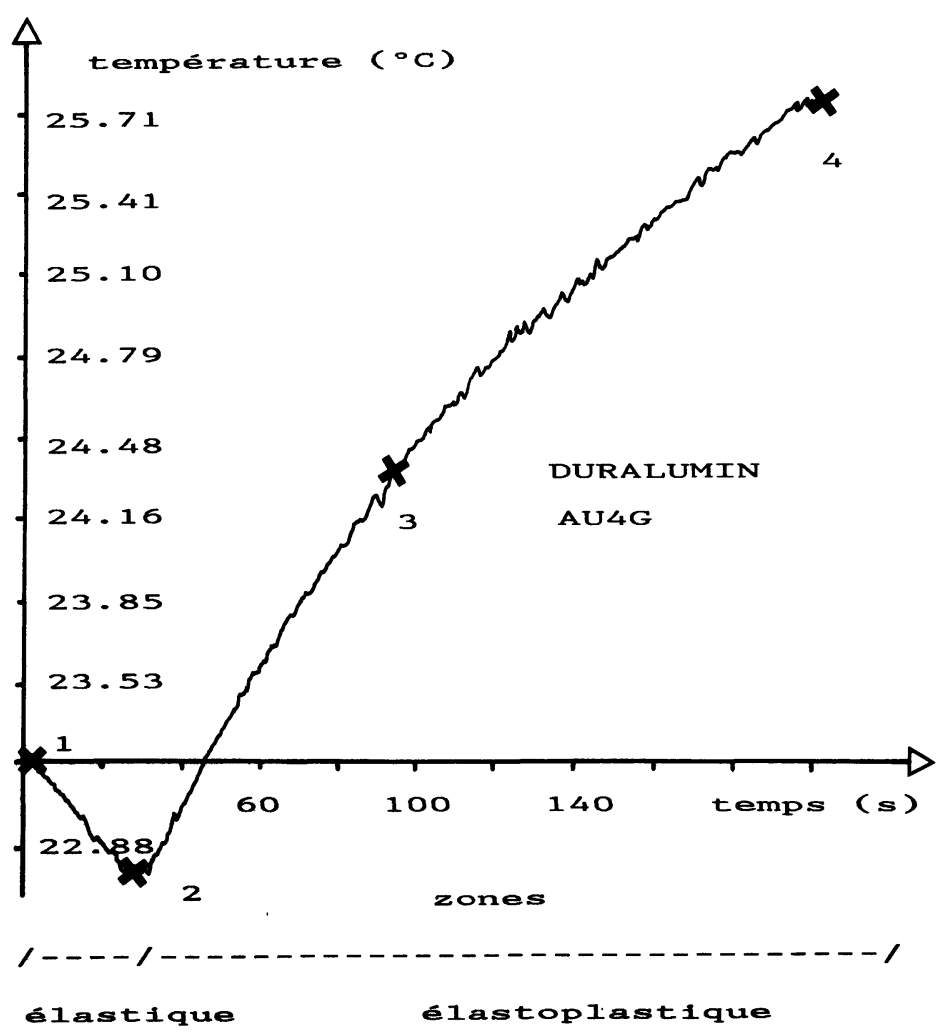

Fig. 2. - Evolution de la température moyenne sur une surface $S$ de l'échantillon; les croix sont associées aux images thermiques (cf. planches 1 à 4).

[Mean temperature evolution on a sample surface $S$; the crosses are associated to thermal pictures, (cf. pictures 1 to 4).]

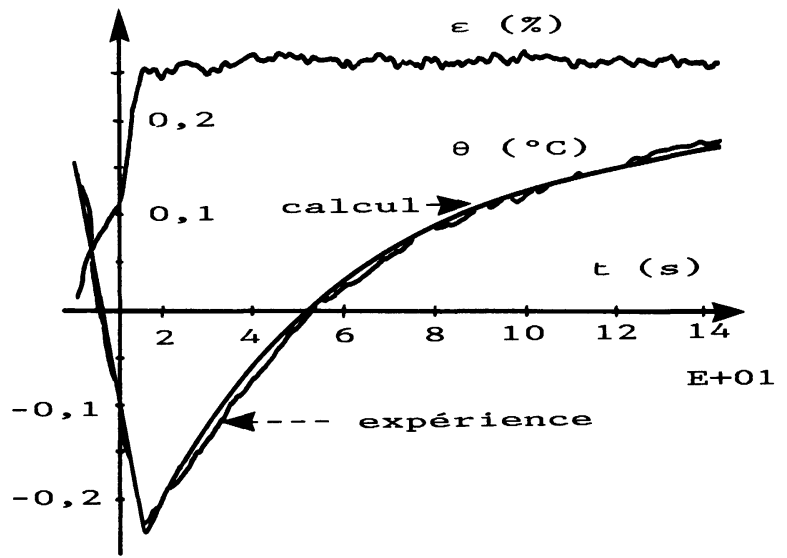

Fig. 3. - Evolution de la température dans le cas d'une charge élastique: comparaison entre la mesure et le calcul ; (AU4G).

[Temperature evolution during elastic load : comparison between measurement and calculation (AU4G).]

\section{Essais, résultats, et discussion.}

Des essais ont été réalisés sur trois matériaux, rencontrés couramment dans l'industrie : un duralumin (hypertrempé), un acier au carbone et un acier inoxydable (trempés vieillis).
Pour que la sollicitation en traction reste homogène dans toute la partie utile de l'éprouvette, on limite la déformation de l'échantillon à $10 \%$ de manière à éviter toute striction locale et en endommagement trop important du matériau. Le module d'Young reste approximativement constant durant de tels processus.

Les mesures « locales » infrarouges sont comparées à celles obtenues globalement par microcalorimétrie [18]. La dispersion des résultats, entre les deux approches, est illustrées par les figures 4 et 5 . Les erreurs relatives, sont de l'ordre de $3,4 \%$ en début d'écrouissage et ne dépassent pas $15 \%$ en fin d'essai.

Les essais réalisés sur les trois matériaux pré-cités montrent que :

- l'évolution de l'énergie bloquée est limitée lors d'écrouissages monotones (Fig. 6) ; le matériau tend asymptotiquement vers un comportement plastique parfait pour lequel toute l'énergie anélastique est dissipée ;

- ceci entraîne une décroissance de la fraction d'énergie bloquée $\left(w_{\mathrm{b}} / w_{\mathrm{a}}\right)$ qui atteint 50 à $60 \%$ en début d'écrouissage (Fig. 7) ;

- en première approximation, l'énergie bloquée évolue linéairement en fonction de la contrainte de 


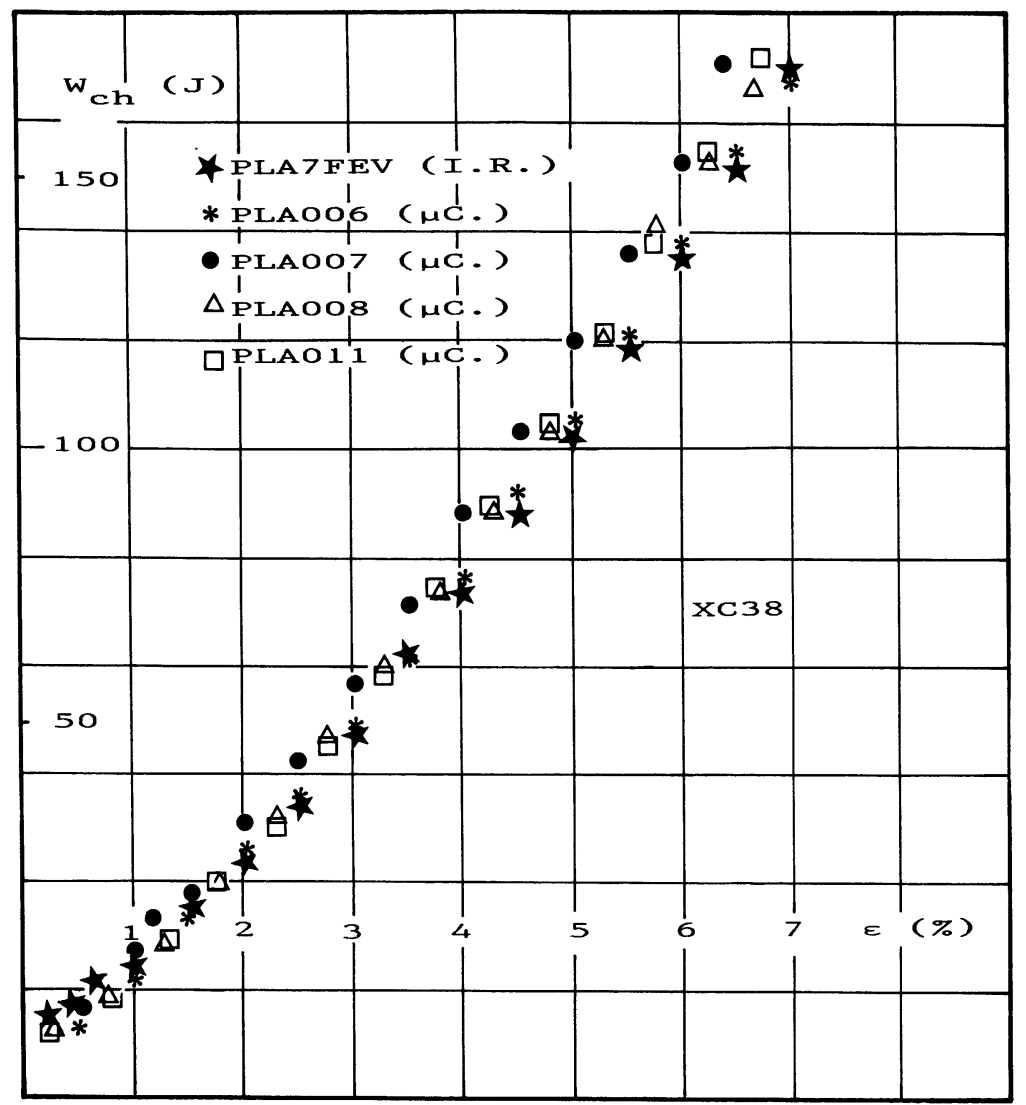

Fig. 4. - Mesures microcalorimétriques et infrarouges de la chaleur dégagée dans un acier au carbone, durant un essai de traction simple.

[Microcalorimetric and infra-red measurements of heat evolved in carbon steel, during tensile test.]

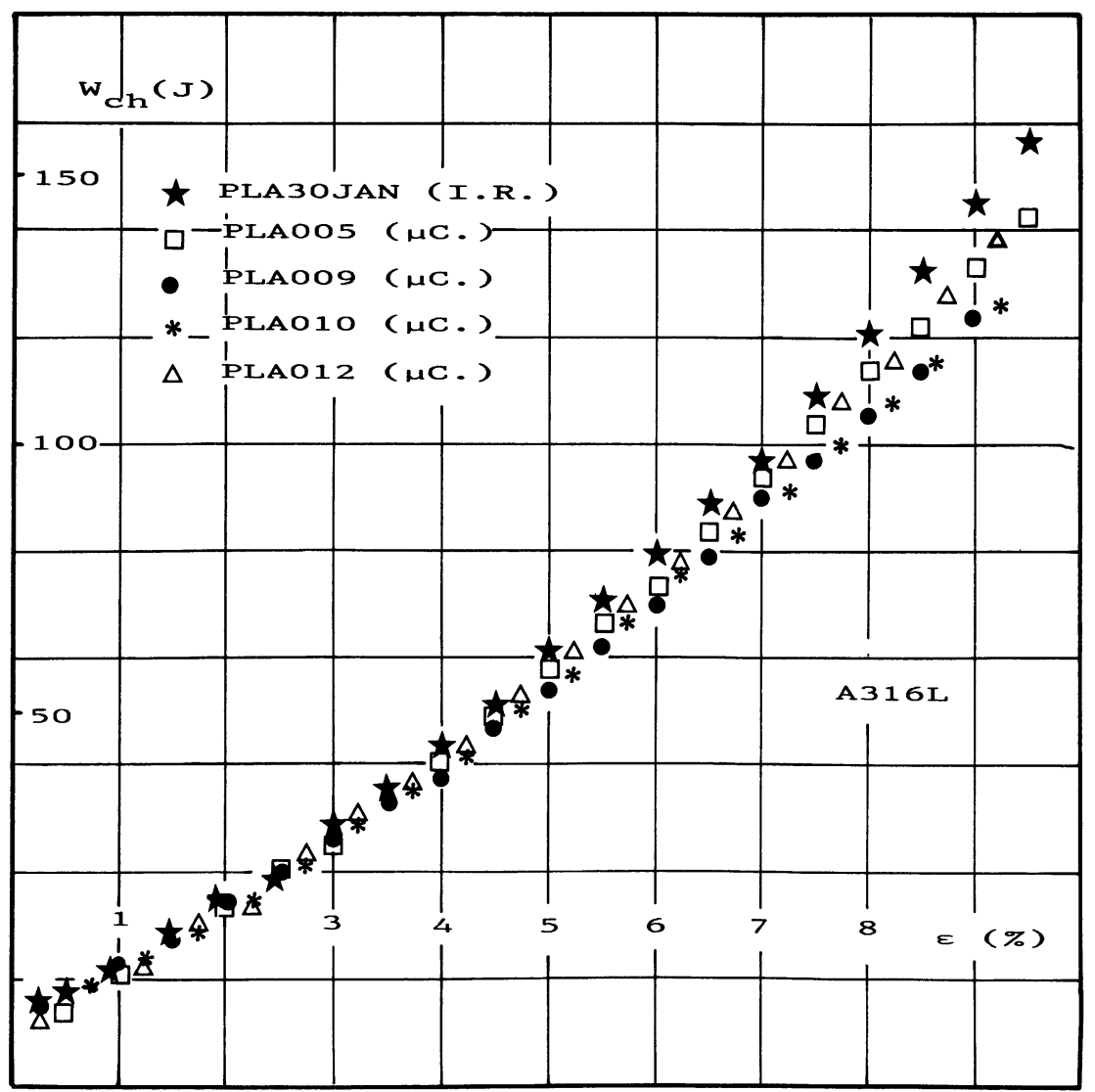

Fig. 5. - Mesures microcalorimétriques et infrarouges de la chaleur dégagée dans un acier inoxydable, durant un essai de traction simple.

[Microcalorimetric and infra-red measurements of heat evolved in stainless steel, during tensile test.] 


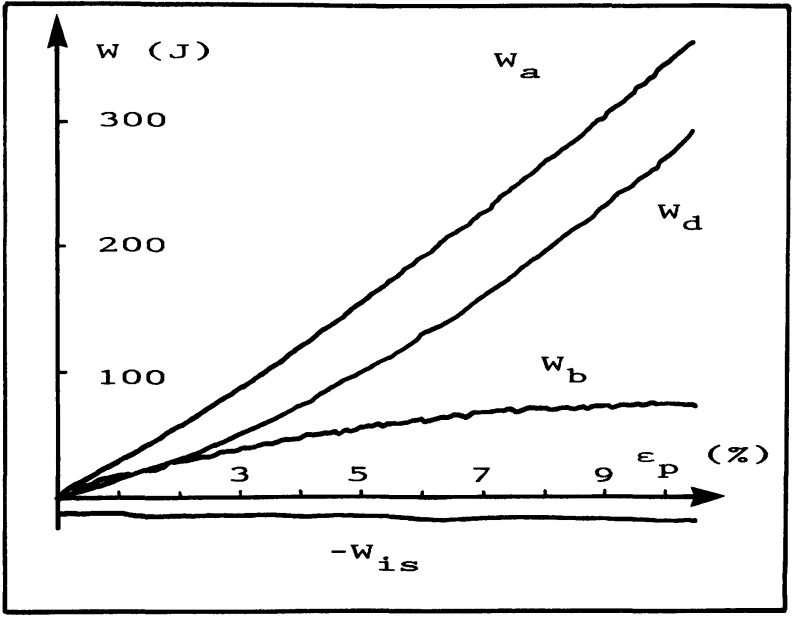

Fig. 6. - Bilan énergétique dans le cas d'un duralumin; limitation de l'énergie bloquée.

[Energy balance in case of duralumin; stored energy limitation.]

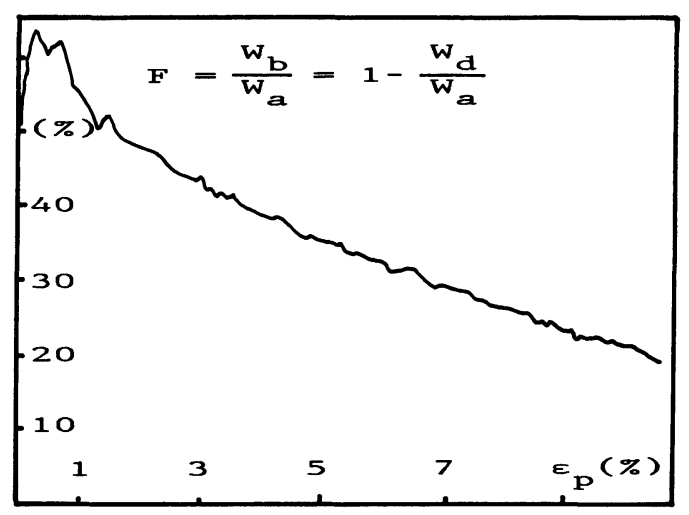

Fig. 7. - Décroissance de la fraction d'énergie bloquée de l'AU4G lors d'un écrouissage monotone.

[Decreasing evolution of the AU4G stored energy ratio during monotonic hardening process.]

traction appliquée, dès que les déformations plastiques apparaissent (Fig. 8).

Si la décroissance de la fraction d'énergie bloquée devient un résultat unanimement reconnu [19], les pourcentages obtenus ici sont beaucoup plus importants que ceux rencontrés dans la littérature (sur des métaux généralement purs et recuits). Les rapports ne dépassent pas $15 \%$, (cf. $[8,19,21]$ ), ce qui explique l'attitude classique consistant à négliger les variations d'énergie interne bloquée lors de l'élaboration de la loi de comportement :

$$
\rho \frac{\partial \psi}{\partial \alpha_{j}} \dot{\alpha}_{j} \ll \sigma: \dot{\varepsilon}_{\mathrm{p}} \quad j=2, \ldots, n
$$

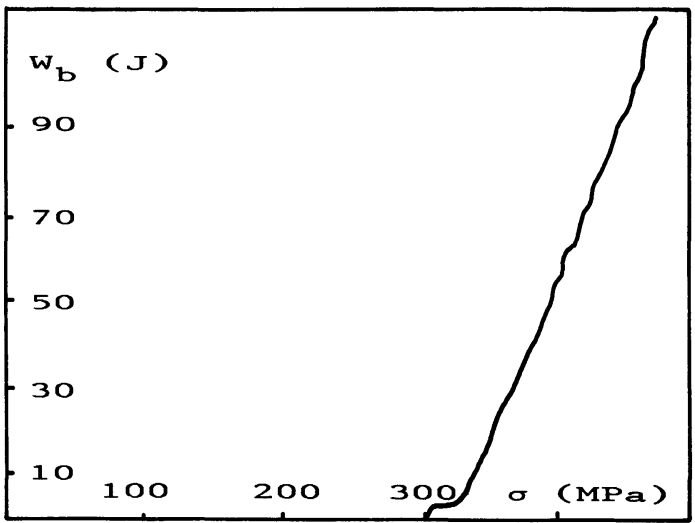

Fig. 8. - Evolution linéaire de l'énergie bloquée avec la contrainte durant un écrouissage monotone ; exemple de l'acier au carbone.

[Linear evolution of the stored energy as a function of stress, during monotonic hardening process; the carbon steel as example.]

Dans le cadre des essais présentés, cette approximation n'est plus justifiée, particulièrement en début d'écrouissage où :

$$
\rho \frac{\partial \psi}{\partial \alpha_{j}} \dot{\alpha}_{j} \approx D_{1} \approx \frac{1}{2} \sigma: \dot{\varepsilon}_{\mathrm{p}} \quad j=2, \ldots, n .
$$

Par ailleurs, on a montré dans [18], que certaines lois classiques ne peuvent qualitativement prévoir la décroissance de la fraction d'énergie bloquée et $a$ fortiori l'évolution limitée de l'énergie bloquée. Par exemple, le modèle de Prandtl-Reuss, fondé sur une hypothèse d'écrouissage isotrope, ou le modèle cinématique linéaire de Prager, ou encore le modèle rhéologique euristique de Saint-Venant généralisé rentrent dans cette catégorie.

En s'inspirant de ces approches classiques, et tenant compte des données énergétiques, de nouvelles lois ont été proposées, [18]. Ces lois rendent compte non seulement de la partie mécanique du phénomène d'écrouissage, mais encore, de l'évolution du bilan énergétique qui lui est associé.

En conclusion, il nous semble donc important d'insister sur le fait que, la connaissance des phénomènes énergétiques associés aux processus quasistatiques de déformation est un atout supplémentaire important, sinon capital pour la détermination des lois de comportement des matériaux solides et ce, même si, les effets thermiques associés sont, eux, négligeables. Les progrès constants réalisés dans le domaine des techniques infrarouges devraient permettre de généraliser ce type d'approche expérimentale à des sollicitations plus complexes que celles réalisées ici, ainsi qu'à l'étude de comportements viscoélastiques ou viscoplastiques. 


\section{Bibliographie}

[1] LoRd Kelvin, Trans. R. Soc. Edimb. 20 (1885) $161 \quad$ [12] Lebon G., MAThieU Ph., J. Entropie 100 (1981) 57-77.

[2] Taylor G. I., Quinney H., Proc. R. Soc. 143A (1934) 307-326.

[3] Buy H. D., Cah. Fran. Rhéo. 1 (1965) 15-19.

[4] Mandel J., Cah. Fran. Rhéo. 1 (1965) 9-13.

[5] Krempl E., Plasticity Today, Eds. A. Sawzuk et G. Bianchi (Els. Sci. Publ.) (1985) 247-257.

[6] Williams R. O., The Review of Scientific Instruments 31 (1960) 1334-1341.

[7] Guillemet et al., J. Phys. Chem. Glasses 6 (1965) 1-5.

[8] Chrysochoos A., Mat. Sci. Eng. (1988) in press.

[9] SaIX C., Jouanna P., J. Méch. Appl. 5 (1981) 457-466.

[10] Bouc R., Nayrolles B., J. Méch. Théo. Appl. 4 (1985) 27-58. 75-85.

[13] Delobelle P., Rev. Phys. Appl. 23 (1988) 1-61.

[14] Chrysochoos A., J. Méch. Théo. Appl. 4 (1985) 589-614.

[15] Bouc R., Nayroles B., J. Méch. Théo. Appl. 4 (1985) 27-58.

[16] BREMOND P., Thèse D.I. (1982) Marseille, France.

[17] GAUSSORGES G., La thermographie infrarouge, Technique et Documentation (1981) Paris.

[18] Chrysochoos A., Thèse d'Etat (1987) Montpellier, France.

[19] Bever M. B., Holt D. L., Titchener A. L., The stored Energy of Cold Work, Pergamonn (1973) New York.

[20] Germain P., Cours de Mécanique des Milieux Continus (1973) Masson, T1, Paris.

[11] Bataille J., Kestin K., J. Méch. 14 (1975) 365-384. [21] Williams R. O., Acta Met. 9 (1961) 949-957. 